\title{
Índice de massa corporal para triagem de fatores de risco para doenças cardiovasculares na infância
}

\author{
Body mass index to the cardiovascular \\ disease risk screening in infancy
}

Gabriel Gustavo Bergmann', Adroaldo Gaya'2, Ricardo Halpern', Mauren Lúcia de Araújo Bergmann 3 , Ricardo Rodrigo Rech ${ }^{4}$, Cristine Boone Constanzi ${ }^{3}$, Lidiane Requia Alli ${ }^{3}$

\author{
Universidade Federal do \\ Pampa (Unipampa), RS, Brasil \\ 2 Universidade Federal do \\ Rio Grande do Sul (UFRGS), \\ Porto Alegre, RS, Brasil \\ ${ }^{3}$ Universidade Luterana do Brasil \\ (Ulbra), Canoas, RS, Brasil \\ ${ }^{4}$ Universidade de Caxias do Sul \\ (UCS), Caxias do Sul, RS, Brasil
}

Correspondência para: Gabriel Gustavo Bergmann Universidade Federal do Pampa BR 472, Km 592, caixa postal 118 97500-970 - Uruguaiana, RS, Brasil gabrielgbergmann@gmail.com

Recebido em 1/Mar/2010 Aceito em 31/Jan/2011

\begin{abstract}
RESUMO
Objetivo: Propor novos pontos de corte para índice de massa corporal (IMC) de escolares de 7 a 12 anos de idade. Sujeitos e métodos: 1.413 escolares de 7 a 12 anos de idade selecionados de forma aleatória. Foram medidos o IMC, o colesterol total (CT) e as pressões arterial sistólica (PAS) e diastólica (PAD). Os dados foram analisados a partir da curva Receiver Operating Characteristic (ROC) e da análise bivariada. Resultados: Os pontos de corte para o IMC propostos no presente estudo apresentam adequada capacidade de identificar verdadeiros positivos e negativos em relação à presença de fatores de risco para doenças cardiovasculares (DCV). Além disso, apresentam valores mais altos e equilibrados de sensibilidade e especificidade que outras propostas de pontos de corte. Conclusões: Os pontos de corte para o IMC propostos no presente estudo configuram-se como a alternativa mais adequada para a triagem de escolares com chances aumentadas de apresentarem fatores de risco para DCV. Arq Bras Endocrinol Metab. 2011;55(2):114-20
\end{abstract}

Descritores

IMC; pontos de corte; escolares; curva ROC

\section{ABSTRACT}

Objective: To purpose news body mass index (BMI) cut offs to 7 to 12 years old scholars. Subjects and methods: 1.413 scholars of 7 to 12 years old selected of aleatory way. Were measured the BMI, total cholesterol (TC), and systolic (SBP) and diastolic blood pressure (DBP). To the date analyze were used the receiver operating characteristic curve (ROC curve) and the bivariate analyze. Results: The BMI cut offs proposed at this study show adequate capacity to identify true positive and negative to the cardiovascular diseases (CVD) risk factors presence. Besides, these cut offs show higher and more balanced sensibility and specificity values than other cut offs proposals. Conclusions: The BMI proposed at this study configure with as the more adequate alternative to the screening for the scholars with increased chances to carry DCV risk factors. Arq Bras Endocrinol Metab. 2011;55(2):114-20

Keywords

BMl; scholars; cut off; ROC curve

\section{INTRODUÇÃO}

A doenças cardiovasculares (DCV) configuram-se como a principal causa de morte no mundo (1). Essas doenças se desenvolvem ao longo da vida de forma lenta e gradual, tendo a infância como ponto de par- tida (2). Entre as variáveis associadas às DCV, o excesso de peso (sobrepeso e obesidade) configura-se como interveniente relevante (3). Como as DCV iniciam seu desenvolvimento na infância, é recomendável que sua prevenção inicie também nesse período, justificando o 
grande número de estudos preocupados com os níveis de excesso de peso em crianças e adolescentes (4).

Entre as alternativas para medidas de excesso de peso em crianças e adolescentes, o índice de massa corporal (IMC) tem se destacado (5). Para a classificação do IMC, os estudos vêm utilizando diferentes propostas de pontos de corte (5). As propostas de Cole e cols. (6) (International Obesity Task Force - IOTF) e de Kuczmarski e cols. (7) (Center of Disease Control -CDC) são as que têm sido utilizadas com maior frequência (811). No Brasil, Conde e Monteiro (12) propuseram a utilização de critérios de classificação do IMC para a população brasileira jovem a partir dos dados do Instituto Nacional de Alimentação e Nutrição (INAM).

A proposta sugerida pelo CDC (7) foi feita com base em uma série de levantamentos sobre saúde da população americana (National Health Examination Surveys - NHANES), realizados de 1963 a 1994. Os pontos de corte para o IMC foram definidos partir dos percentis 5 (baixo peso), 85 (risco de sobrepeso) e 95 (sobrepeso) por sexo e idade. A proposição dos pontos de corte do IOTF (6) e de Conde e Monteiro (12) foi feita levando em consideração os valores de $25 \mathrm{~kg} / \mathrm{m}^{2}$ para sobrepeso e $30 \mathrm{~kg} / \mathrm{m}^{2}$ para obesidade em adultos, e identificando, a partir da técnica curvas suavizadas específicas para a idade (LMS), em qual percentil esses valores aconteciam aos 18 anos, mantendo-se tal percentil para as idades inferiores como os pontos de corte.

Alguns estudos objetivaram identificar a validade dos pontos de corte propostos na literatura para o IMC como indicador de excesso de peso utilizando o percentual de gordura como referência $(13,14)$. Os resultados indicaram que, em geral, os pontos de corte para o IMC de crianças e adolescentes propostos pela literatura apresentam adequada concordância com a adiposidade. Contudo, algumas considerações devem ser feitas. Do ponto de vista metodológico, as propostas foram baseadas nos valores que em adultos apresentam maiores chances de desenvolvimento de $\operatorname{DCV}(6,12)$, ou em escolhas arbitrárias na distribuição dos valores do IMC da população (7). Mesmo com adequada concordância com a adiposidade, os valores dos pontos de corte desses estudos não garantem que indivíduos que ultrapassam seus valores tenham maior probabilidade de apresentar fatores de risco para DCV. Nesse contexto, três estudos nacionais propuseram valores de ponto de corte a partir dos resultados da curva ROC entre o IMC e fatores de risco para DCV (15-17). Contudo, nenhum dos três estudos propôs valores de ponto de corte para diferentes idades, apresentando dessa forma limitações para utilização em grupos com idades variadas.

Diante desse quadro, torna-se importante o desenvolvimento de propostas de pontos de corte para o IMC por sexo e idade que utilizem como referência fatores de risco para DCV, constituindo-se como alternativas práticas de diagnóstico para a triagem de escolares com chances aumentadas de apresentarem fatores de risco para DCV. Diante dessas considerações, os objetivos do presente estudo são: a) propor pontos de corte para o IMC de escolares de 7 a 12 anos de idade; b) avaliar o desempenho de pontos de corte para o IMC propostos pela literatura como instrumento de triagem na identificação de escolares com fatores de risco para DCV, e; c) identificar qual das propostas de ponto de corte para o IMC apresenta a maior capacidade na identificação de escolares com fatores de risco para DCV.

\section{SUJEITOS E MÉTODOS}

Este estudo de corte transversal de base escolar foi realizado com escolares de 7 a 12 anos de idade dos dois sexos matriculados nas redes de ensino municipal, estadual e particular da cidade de Caxias do Sul, RS. A população de escolares de 7 a 12 anos de idade no ano de 2005, de acordo com dados da Secretaria Estadual de Educação, foi de 33.241 escolares. Foi estimada para o cálculo de tamanho da amostra uma prevalência média de sobrepeso/obesidade de $20 \%$ (11). Com um intervalo de confiança de $95 \%$, um poder de $80 \%$ e um erro de estimativa de 3\% seriam necessárias 669 crianças. Utilizando-se um efeito de delineamento 2 para controle de fatores de confusão, acrescido de mais $15 \%$ para suprir possíveis perdas e recusas, foi estimada a necessidade de avaliar 1.573 crianças. Das 1.573 crianças estimadas para o estudo, 1.413 realizaram as medidas de IMC e pressão arterial (PA); 1.294 permitiram a realização da coleta de sangue. O critério de amostragem adotado foi probabilístico por conglomerados, em que cada escola foi considerada um conglomerado. Todas as escolas do município participaram do sorteio tendo as mesmas chances de participarem do estudo de acordo com o número de alunos matriculados na faixa etária de 7 a 12 anos. Todos os escolares que participaram da composição da amostra apresentaram o termo de consentimento livre e esclarecido. O estudo foi aprovado pelo Comitê de Ética da Universidade Luterana do Brasil (protocolo 2006-365H). Os dados foram coletados de abril a agosto de 2007.

O IMC foi obtido por meio da divisão da massa corporal (quilogramas) pela estatura (metros) ao quadra- 
do. Massa corporal e estatura foram medidas a partir de orientações do PROESP-BR (18). Para a análise por critérios de referência foram utilizados os pontos de corte propostos por Cole e cols. (6), Kuczmarski e cols. (7) e por Conde e Monteiro (12).

Os fatores de risco para DCV medidos nos escolares foram o colesterol total (CT), a pressão arterial sistólica (PAS) e a pressão arterial diastólica (PAD). Os valores de CT foram obtidos mediante utilização do monitor portátil Accutrend ${ }^{\circledR}$ GCT (Roche Diagnostics). Não foi exigido que os escolares estivessem em jejum, já que a o CT não apresenta variação significativa com o indivíduo estando ou não em jejum (19). A utilização dessa forma de medida do CT (monitor portátil Accutrend ${ }^{\circledR}$ GCT Roche Diagnostics) e desse procedimento (não jejum) foi utilizada em campanha no Brasil pela Sociedade Brasileira de Cardiologia em mais de 81 mil indivíduos (20). Foi considerado CT “desejável” valores menores que $170 \mathrm{mg} / \mathrm{dl}$, CT "limítrofe" valores entre 170 e 199 mg/dl e CT "aumentado" valores iguais ou maiores que $200 \mathrm{mg} / \mathrm{dl}$ (19).

As medidas da PA foram realizadas em uma sala onde três avaliadores previamente treinados e capacitados para a coleta se dispunham separadamente uns dos outros. Os estetoscópios (pediátricos) e os manguitos (pediátricos) eram da marca Becton Dickinson ${ }^{\circledR} \mathrm{e}$ os esfigmomanômetros eram das marcas Cardiomed ${ }^{\circledR} \mathrm{e}$ Oxigen $^{\circledR}$, todos aferidos pelo Instituto Nacional de Metrologia. A PAS foi determinada no aparecimento do primeiro som de Korotkof e a PAD, no desaparecimento deste. Foram realizadas três medidas com intervalos de 3 minutos. Para a categorização da pressão arterial, foi considerado o sexo, a idade e o percentil da estatura. Foram considerados "normais" indivíduos com PAS e PAD inferiores ao percentil 90; "pré-hipertensos" entre percentis 90 e 95; e "hipertensos" com percentil acima de 95 (21).

Com relação ao tratamento dos dados, para a proposição dos pontos de corte para o IMC foi utilizada a curva Receiver Operating Characteristic (ROC). Esse procedimento necessita de uma variável "referência" (variável dicotômica) e uma variável "teste" (no caso do presente estudo o IMC). Para tanto foram criadas nove possibilidades de referência a partir dos fatores de risco para DCV dicotomizados da seguinte maneira: 1 ) CT “desejável/limítrofe" e "aumentado"; 2) CT “desejável" e "limítrofe/aumentado"; 3) PAS "normal/ pré-hipertenso" e "hipertenso"; 4) PAS "normal" e "pré-hipertenso/hipertenso"; 5) PAD "normal/pré- -hipertenso" e "hipertenso"; 6) PAD "normal" "pré-hipertenso/hipertenso"; 7) escore de fatores de risco para DCV $=5($ escore DCV $\geq 5) ; 8)$ escore DCV $\geq 6$, e; 9) escore DCV $\geq 7$. Os três escores de fatores de risco para DCV foram criados a partir de um somatório que levou em consideração a classificação do CT pelas III DBSD (19), e da PAS e PAD pela NHBPEP (21), em que a inexistência do fator de risco $(\mathrm{CT}=$ desejável; PAS e PAD = normal) recebeu o valor " 1 ", a existência da classificação intermediária do fator de risco $(\mathrm{CT}=$ limítrofe; PAS e PAD = pré-hipertenso $)$ recebeu o valor "2", e a existência do fator de risco $(\mathrm{CT}=$ aumentado; PAS e PAD = hipertenso $)$ recebeu o valor " 3 ". Após a criação das nove referências de fatores de risco para DCV, foi determinada a curva ROC entre cada uma delas e o IMC estratificado por sexo e idade. A referência que na média entre as idades apresentou a maior área sob a curva ROC foi escolhida como a referência de fatores de risco para DCV. Para os meninos a referência foi o escore DCV $\geq 6(0,802)$ e para as meninas o escore DCV $\geq 7(0,741)$. Com as referências para os fatores de risco para DCV definidas, foram identificados os valores específicos de IMC em cada idade e nos dois sexos que apresentaram o melhor ajustamento entre sensibilidade e especificidade, identificando, assim, os pontos de corte para o IMC a partir dos valores apresentados por nossa amostra. A análise quanto à validade dos pontos de corte para o IMC sugeridos pela literatura e pela amostra do presente estudo na tentativa de identificar indivíduos com chances aumentadas de apresentar fatores de risco para as DCV foi realizada com base em uma análise bivariada entre o IMC dos escolares categorizados por cada uma das propostas de pontos de corte e as referências que apresentaram a maior área sob a curva ROC seguidos dos cálculos de sensibilidade e especificidade. Com o intuito de identificar quanto os indivíduos que ultrapassaram o ponto de corte para o IMC em cada uma das propostas estudadas apresentam a mais de chance de portarem fatores de risco para as DCV que seus pares que não ultrapassaram o valor do ponto de corte, foi utilizada análise bivariada seguida do cálculo de razão de chances (Odds Ratio - OD). As análises para a identificação dos pontos de corte foram realizadas levando-se em consideração uma área sob a curva ROC mínima de $50 \%(22)$ e as análises bivariadas, um intervalo de confiança de 95\% (IC 95\%) e P $\leq 0,05$. Todas as análises foram realizadas no programa SPSS for Windows versão 13.0. 


\section{RESULTADOS}

Os resultados da análise da curva ROC entre o IMC e as referências de fatores de risco para DCV por sexo e idade estão apresentadas na tabela 1 . Analisando os resultados, percebe-se que os valores propostos como pontos de corte são crescentes ao longo das idades para meninos e meninas. A área sob a curva ROC ultrapassou os $50 \%$ em todas as idades e nos dois sexos. Os valores de sensibilidade variaram de $0,600(60 \%)$ a $1,00(100 \%)$, demonstrando adequada capacidade de identificar escolares classificados como portadores de fatores de risco para DCV que ultrapassaram os valores dos pontos de corte (verdadeiros positivos). Os valores de especificidade variaram de $0,519(51,9 \%)$ a 0,898 $(89,8 \%)$, demonstrando adequada capacidade de identificar escolares classificados como não portadores de fatores de risco para DCV que não ultrapassaram os valores dos pontos de corte (verdadeiros negativos).

Na tabela 2 são apresentados os valores de sensibilidade e especificidade calculados com base na associação entre os pontos de corte para o IMC propostos por Cole e cols. (6), por Kuczmarski e cols. (7), por Conde e Monteiro (12) e pelo presente estudo com as referências de fatores de risco para DCV estratificados por sexo. Analisando os resultados, nota-se que a proposta que apresenta os valores mais altos e com melhor equi- líbrio entre sensibilidade e especificidade é a do presente estudo (Tabela 2).

Quando o IMC é categorizado pelos diferentes pontos de corte propostos e associado às referências de fatores de risco para DCV seguido do cálculo da razão de chances $(\mathrm{OR})$, percebe-se que independentemente da proposta, para os dois sexos, os escolares que ultrapassaram os valores dos pontos de corte apresentam mais chances de portarem fatores de risco para DCV (Tabela 3). Entre as propostas de pontos de corte para o IMC, a que apresentou maiores chances (OR) para os escolares que ultrapassaram os valores de ponto de corte de portarem fatores de risco para DCV foi a do presente estudo (Tabela 3 ).

\section{DISCUSSÃO}

Considerando as evidências de que indivíduos com IMC elevados estão mais suscetíveis a desenvolver DCV e seus fatores de risco (3) e que tais doenças iniciam na infância e adolescência (2), identificar valores que aumentam as chances de escolares desenvolverem fatores de risco para DCV parece fundamental, constituindo-se como instrumento para prevenção primária. Dessa forma, o presente estudo configura-se como a primeira tentativa nacional de proposição de pontos de corte

Tabela 1. Resultados da curva ROC entre o IMC e as referências de fatores de risco para DCV

\begin{tabular}{|c|c|c|c|c|c|c|c|c|c|c|c|c|}
\hline \multirow{2}{*}{ Idade } & \multicolumn{6}{|c|}{ Meninos (escore DCV $\geq 6$ ) } & \multicolumn{6}{|c|}{ Meninas (escore DCV $\geq 7$ ) } \\
\hline & $\mathbf{n}$ & ASC & IC (95\%) & SENS & ESP & PC & $\mathbf{n}$ & ASC & IC (95\%) & SENS & ESP & PC \\
\hline 7 anos & 61 & 0,69 & $0,56-0,83$ & 0,66 & 0,67 & 17,86 & 56 & 0,67 & $0,53-0,80$ & 0,50 & 0,71 & 17,16 \\
\hline 8 anos & 116 & 0,93 & $0,85-1,00$ & 1,00 & 0,89 & 19,26 & 108 & 0,61 & $0,31-0,91$ & 0,40 & 0,70 & 18,22 \\
\hline 9 anos & 140 & 0,72 & $0,58-0,88$ & 0,66 & 0,70 & 19,38 & 111 & 0,78 & $0,60-0,96$ & 0,66 & 0,85 & 19,18 \\
\hline 10 anos & 130 & 0,87 & $0,74-1,00$ & 0,85 & 0,89 & 20,74 & 126 & 0,67 & $0,43-0,92$ & 0,60 & 0,76 & 20,92 \\
\hline 11 anos & 118 & 0,74 & $0,52-0,96$ & 0,66 & 0,77 & 22,16 & 137 & 0,89 & $0,80-0,99$ & 0,80 & 0,80 & 22,39 \\
\hline 12 anos & 80 & 0,84 & $0,75-0,94$ & 0,80 & 0,81 & 22,23 & 87 & 0,80 & $0,65-0,95$ & 0,75 & 0,79 & 22,66 \\
\hline
\end{tabular}

n: amostra; ASC: área sob a curva ROC; IC (95\%): intervalo de confiança de 95\%; SENS: sensibilidade; ESP: especificidade; PC: ponto de corte referente ao IMC (Kg/m²) para 0 avaliado apresentar chances aumentadas de portar fatores de risco para DCV.

Tabela 2. Sensibilidade e especificidade dos pontos de corte para o IMC propostos por Cole e cols. (6), por Kuczmarski e cols. (7), por Conde e Monteiro (12) e pelo presente estudo em relação às referências de fatores de risco para DCV

\begin{tabular}{|c|c|c|c|c|}
\hline \multirow{2}{*}{$\begin{array}{l}\text { PC } \\
\text { sugeridos }\end{array}$} & \multicolumn{2}{|c|}{ Meninos escore de DCV $\geq 6$} & \multicolumn{2}{|c|}{ Meninas escore de DCV $\geq 7$} \\
\hline & Sens (IC 95\%) & Esp (IC 95\%) & Sens (IC 95\%) & Esp (IC 95\%) \\
\hline Cole e cols. (2) & $0,61(0,46-0,76)$ & $0,74(0,70-0,78)$ & $0,55(0,36-0,74)$ & $0,73(0,69-0,77)$ \\
\hline Kuczmarski e cols. (16) & $0,68(0,53-0,83)$ & $0,70(0,66-0,74)$ & $0,59(0,41-0,77)$ & $0,73(0,69-0,77)$ \\
\hline Conde e Monteiro (3) & $0,68(0,53-0,83)$ & $0,69(0,65-0,73)$ & $0,65(0,47-0,83)$ & $0,64(0,60-0,68)$ \\
\hline Presente estudo & $0,73(0,59-0,87)$ & $0,78(0,75-0,81)$ & $0,69(0,52-0,86)$ & $0,75(0,71-0,79)$ \\
\hline
\end{tabular}

PC: pontos de corte; sens: sensibilidade; esp: especificidade; IC 95\%: intervalo de confiança de $95 \%$. 
para o IMC, por sexo e idade, em uma amostra populacional de base escolar que apresente validação empírica pela associação com fatores de risco para DCV.

Os resultados encontrados referentes à proposição de pontos de corte para o IMC a partir dos valores da amostra do presente estudo com os escores de fatores de risco para DCV demonstraram valores de área sob a curva ROC, de sensibilidade e especificidade similares aos reportados por outros estudos que propuseram pontos de corte para o IMC utilizando fatores de risco para DCV como referência (15-17). Guedes e cols. (15) propuseram pontos de corte para o IMC em uma amostra de 281 adolescentes entre 15 e 18 anos mediante análise da curva ROC utilizando como referência um escore feito a partir dos valores do percentil 75 de fatores de risco para DCV. Em estudo realizado com 374 escolares com idade média de 11 anos, Lunardi e
Petroski (16) utilizaram lípides sanguíneos para propor pontos de corte para o IMC. Utilizando também lípides sanguíneos e glicose plasmática, Vieira e cols. (17) propuseram pontos de corte para o IMC a partir de informações de 577 adolescentes de 12 a 19 anos de idade.

Os três estudos, assim como o presente estudo, encontraram valores de área sob a curva ROC, de sensibilidade e especificidade que permitem a utilização dos pontos de corte propostos como instrumento de triagem de crianças e adolescentes com maiores chances de apresentarem fatores de risco para DCV (Tabela 4). Contudo, é importante destacar que apenas o presente estudo propõe pontos de corte específico por sexo e idade. Os demais apresentam como limitação o fato de proporem valores de ponto de corte para o IMC estratificado apenas por sexo, não considerando valores específicos por idade (Tabela 4 ).

Tabela 3. Razão de chances (OD) entre as referências de fatores de risco para DCV e o IMC categorizado pelas diferentes propostas de pontos de corte

\begin{tabular}{|c|c|c|c|c|c|c|c|c|}
\hline \multirow{2}{*}{$\begin{array}{l}\text { PC } \\
\text { sugeridos }\end{array}$} & \multicolumn{4}{|c|}{ Meninos (escore DCV $\geq 6$ ) } & \multicolumn{4}{|c|}{ Meninas (escore DCV $\geq 7$ ) } \\
\hline & n (\%) & $\mathbf{O R}$ & IC (95\%) & $\mathbf{P}$ & n (\%) & $\mathbf{O R}$ & IC (95\%) & $\mathbf{P}$ \\
\hline \multicolumn{9}{|l|}{ Cole e cols. (6) } \\
\hline Não ultrapassaram o PC & $463(71,8)$ & 1,00 & - & - & $452(72,3)$ & 1,00 & - & - \\
\hline Ultrapassaram o PC & $182(28,2)$ & 4,44 & $2,31-8,55$ & 0,000 & $173(27,7)$ & 3,44 & $1,61-7,31$ & 0,000 \\
\hline \multicolumn{9}{|l|}{ Kuczmarski e cols. (7) } \\
\hline Não ultrapassaram o PC & $437(67,8)$ & 1,00 & - & - & $448(71,7)$ & 1,00 & - & - \\
\hline Ultrapassaram o PC & $208(32,2)$ & 5,07 & $2,56-10,02$ & 0,000 & $177(28,3)$ & 3,86 & $1,80-8,26$ & 0,000 \\
\hline \multicolumn{9}{|l|}{ Conde e Monteiro (12) } \\
\hline Não ultrapassaram o PC & $429(66,5)$ & 1,00 & - & - & $393(62,9)$ & 1,00 & - & - \\
\hline Ultrapassaram o PC & $216(33,5)$ & 4,76 & $2,41-9,40$ & 0,000 & $232(37,1)$ & 3,41 & $1,56-7,48$ & 0,000 \\
\hline \multicolumn{9}{|l|}{ Presente estudo } \\
\hline Não ultrapassaram o PC & $482(74,7)$ & 1,00 & - & - & $455(72,8)$ & 1,00 & - & - \\
\hline Ultrapassaram o PC & $163(25,3)$ & 9,65 & $4,71-19,78$ & 0,000 & $170(27,2)$ & 6,60 & $2,94-14,82$ & 0,000 \\
\hline
\end{tabular}

n(\%): número amostral absoluto e (percentual); OR: razão de chance; IC(95\%): intervalo de confiança de 95\%; P: significância.

Tabela 4. Resultados da análise da curva ROC entre o IMC e fatores de risco para DCV dos estudos de Guedes e cols. (15), Lunardi e Petroski (16), Vieira e cols. (17) e do presente estudo

\begin{tabular}{|c|c|c|c|c|c|c|c|c|c|c|c|c|c|}
\hline & \multirow[b]{2}{*}{ Idade } & \multicolumn{6}{|c|}{ Meninos } & \multicolumn{6}{|c|}{ Meninas } \\
\hline & & $\mathbf{n}$ & ASC & IC (95\%) & SENS & ESP & PC & $\mathbf{n}$ & ASC & IC (95\%) & SENS & ESP & PC \\
\hline Guedes e cols. (15) & 15-18 anos & 157 & 0,77 & $0,69-0,84$ & 53 & 85 & 22 & 124 & 0,76 & $0,68-0,83$ & 54 & 81 & 23 \\
\hline Lunardi e Petroski (16) & 11 anos & 166 & 0,87 & $0,78-0,95$ & 0,83 & 67,5 & 19,3 & 208 & 0,74 & $0,63-0,86$ & 0,71 & 0,73 & 19,3 \\
\hline Vieira e cols. (17) & 12-19 anos & 210 & 0,67 & $0,52-0,81$ & 0,62 & 0,61 & 20,4 & 367 & 0,65 & $0,53-0,76$ & 0,66 & 0,66 & 20,3 \\
\hline \multirow[t]{6}{*}{ Presente estudo } & 7 anos & 61 & 0,69 & $0,56-0,83$ & 0,66 & 0,67 & 17,86 & 56 & 0,67 & $0,53-0,80$ & 0,50 & 0,71 & 17,16 \\
\hline & 8 anos & 116 & 0,93 & $0,85-1,00$ & 1,00 & 0,89 & 19,26 & 108 & 0,61 & $0,30-0,91$ & 0,40 & 0,70 & 18,22 \\
\hline & 9 anos & 140 & 0,72 & $0,58-0,88$ & 0,66 & 0,70 & 19,38 & 111 & 0,78 & $0,60-0,96$ & 0,66 & 0,85 & 19,18 \\
\hline & 10 anos & 130 & 0,87 & $0,90-1,00$ & 0,85 & 0,89 & 20,74 & 126 & 0,67 & $0,43-0,92$ & 0,60 & 0,76 & 20,92 \\
\hline & 11 anos & 118 & 0,74 & $0,30-0,96$ & 0,66 & 0,77 & 22,16 & 137 & 0,89 & $0,80-0,99$ & 0,80 & 0,80 & 22,39 \\
\hline & 12 anos & 80 & 0,84 & $0,75-0,94$ & 0,80 & 0,81 & 22,23 & 87 & 0,80 & $0,65-0,95$ & 0,75 & 0,79 & 22,66 \\
\hline
\end{tabular}

n: amostra; ASC: área sob a curva ROC; IC(95\%): intervalo de confiança de 95\%; SENS: sensibilidade; ESP: especificidade; PC: ponto de corte; PC: ponto de corte. 
Com relação às análises quanto à validade dos pontos de corte para o IMC propostos por Cole e cols. (6), por Kuczmarski e cols. (7), e por Conde e Monteiro (12), os resultados de sensibilidade e especificidade encontrados apresentaram valores que podem ser considerados satisfatórios para triagem de escolares com risco aumentado para a presença de fatores de risco para DCV. Resultados prévios corroboram com os achados do presente estudo, indicando que tais propostas de pontos de corte para o IMC apresentam adequada capacidade de identificar indivíduos portadores de fatores de risco para DCV (9). Contudo, entre os pontos de corte estudados, os propostos no presente estudo foram aqueles que melhores ajustamentos entre sensibilidade e especificidade apresentaram, indicando essa proposta como a mais indicada para discriminar escolares com maiores chances de portarem e desenvolverem fatores de risco para DCV (Tabela 3 ).

Os valores encontrados de área sob a curva ROC, sensibilidade e especificidade neste estudo a partir dos valores de IMC e das referências de fatores de risco para DCV talvez não tenham sido ainda mais elevados por haver uma série de outras variáveis que contribuem para a variação dos resultados dos fatores de risco para $\mathrm{DCV}$, além do IMC, que não foram controlados. Entre essas variáveis, podemos destacar o histórico familiar, o estágio puberal, o nível de aptidão física, o nível de atividade física habitual, os hábitos alimentares, e o nível socioeconômico $(8,11,23-27)$. Todavia, por terem sido encontrados satisfatórios valores de área sob a curva ROC, de sensibilidade e especificidade, tal restrição não invalida os resultados encontrados como propostas para pontos de corte para o IMC de escolares.

A possibilidade de utilização dos pontos de corte para o IMC propostos no presente estudo indicada por apresentar o melhor equilíbrio entre sensibilidade e especificidade entre os pontos de corte estudados fica reforçada pelos resultados disponibilizados pela análise bivariada e o cálculo da razão de chances. Essa análise identificou que escolares que ultrapassaram os pontos de corte propostos possuem mais chance $(\mathrm{OR}=9,65$; IC $=4,71-19,78-$ meninos; $\mathrm{OR}=6,60 ; \mathrm{IC}=2,94-14,82$ - meninas) de apresentarem fatores de risco para DCV que aqueles que não ultrapassaram. Esses resultados vão ao encontro de evidências disponíveis na literatura que indicam associação direta entre valores elevados de IMC e a presença de fatores de risco para DCV $(10,23,25,26)$.

Diante dos resultados encontrados ficam evidências de que indivíduos com valores elevados de IMC possuem chances aumentadas de apresentar fatores de risco para DCV em comparação com aqueles com valores mais adequados. Além disso, os pontos de corte propostos no presente estudo mostraram-se mais adequados que aqueles disponíveis na literatura para a triagem de escolares com maiores chances de apresentarem fatores de risco para DCV. Dessa forma, sugerem-se a medida de IMC e a avaliação pelos pontos de corte propostos no presente estudo para triagem de escolares com chances aumentadas para apresentarem fatores de risco para DCV.

Declaração: os autores declaram não haver conflitos de interesse científico neste estudo.

\section{REFERÊNCIAS}

1. WHO. World Health Organization. The Global Burden of Disease: 2004 UpDate. WHO Press: Geneva; 2008.

2. Raitakari OT, Juonala M, Kähönen M, Taittonen L, Tomi Laitinen T, Mäki-Torkko N, et al. Cardiovascular risk factors in childhood and carotid artery intima-media thickness in adulthood: the cardiovascular risk in young finns study. JAMA. 2003;290(17):2277-83.

3. Kenchaiah S, Ace E, Levy D, Wilson PWF, Benjamin EJ, Larson $\mathrm{Mg}$, et al. Obesity and the risk of heart failure. $\mathrm{N}$ Engl $\mathrm{J}$ Med. 2002;347(5):305-13.

4. Janssen I, Katzmarzyk PT, Boyce WF, Vereecken C, Mulvihill C, Roberts $C$, et al. Comparison of overweight and obesity prevalence in school-aged youth from 34 countries and their relationships with physical activity and dietary patterns. Obes Rev. 2005;6(2):123-32.

5. Neovius MG, Linné Y, Barkeling B, Rössner S. Discrepancies between classification systems of childhood obesity. Obes Rev. 2004;5(2):105-14.

6. ColeTJ, Bellizzi MC, Flegal KM, Dietz WH. Establishing a standard definition of child overweight and obesity worldwide: international survey. BMJ. 2000;320(7244):1240-3.

7. Kuczmarski RJ, Ogden CL, Grummer-Strawn LM, Flegal KM, Guo SS, et al. 2000 CDC growth charts: United States. Vital and Health Statistics. Advance Data. 2002(314):1-28.

8. Farias Jr JC, Silva KS. Sobrepeso/obesidade em adolescentes escolares da cidade de João Pessoa, PB: prevalência e associação com fatores demográficos e socioeconômicos. Rev Bras Med Esporte. 2008;14(2):104-8.

9. Freedman DS, Kahn HS, Mei Z, Grummer-Strawn LM, Dietz WH, Srinivasan $S R$, et al. Relation of body mass index and waist-to-height ratio to cardiovascular disease risk factors in children and adolescents: the Bogalusa Heart Study. Am J Clin Nutr. 2007;86(1):33-40.

10. Guimarães ICB, Almeida AM, Santos AS, Barbosa DBV, Guimarães $A C$. Pressão arterial: efeito do índice de massa corporal e da circunferência abdominal em adolescentes. Arq Bras Cardiol. 2008;90(6):393-9.

11. Terres NG, Pinheiro RT, Horta BL, Pinheiro KAT, Horta LL. Prevalência e fatores associados ao sobrepeso e à obesidade em adolescentes. Rev Saúde Pública. 2006;40(4):1-7.

12. Conde WL, Monteiro CA. Valores críticos do índice de massa corporal para classificação do estado nutricional de crianças e adolescentes brasileiros. J Pediatr. 2006;82(4):266-72.

13. Farias Jr JC, Konrad LM, Rabacow FM, Grup S, Araújo VC. Sensibilidade e especificidade de critérios de classificação do ín- 
dice de massa corporal em adolescentes. Rev Saúde Pública. 2009;43(1):53-9.

14. Vitolo MR, Campagnolo PDB, Barros ML, Gama CM, Lopez FA. Avaliação de duas classificações para excesso de peso em adolescentes brasileiros. Rev Saúde Pública. 2007;41(4):653-6.

15. Guedes DP, Guedes JERP, Barbosa DS, Oliveira JA. Aptidão física relacionada à saúde e fatores de risco predisponentes às doenças cardiovasculares em adolescentes. Rev Port Cien Desp. 2002;2(5):31-46.

16. Lunardi CC, Petroski EL. Índice de massa corporal, circunferência da cintura e dobra cutânea triciptal na predição de alterações lipídicas em crianças com 11 anos de idade. Arq Bras Endocrinol Metab. 2008;52(6):1009-14.

17. Vieira ACR, Alvarez MM, Salim Kanaan S, Sichieri R, Gloria V, Veiga GV. Body mass index for predicting hyperglycemia and serum lipid changes in Brazilian adolescents. Rev Saúde Pública. 2009;43(1):44-52.

18. Gaya A, Silva GMG. PROESP-BR - Observatório permanente dos indicadores de saúde e fatores de prestação esportiva em crianças e jovens. Manual de aplicação de medidas e testes, normas e critérios de avaliação; 2007. Disponível em: http://www.proesp. ufrgs.br. Acesso em: Out 20, 2007.

19. III Diretrizes brasileiras sobre dislipidemias e diretriz de prevenção da aterosclerose do Departamento de Aterosclerose da Sociedade Brasileira de Cardiologia. Arq Bras Cardiol. 2001;77(Supl III):1-48.

20. Martinez TL, Rocha SRD, Armaganijan D, Torres KP, Loures-Vale A, Magalhães ME, et al. National alert campaign about increased cholesterol: determination of cholesterol levels in 81,262 Brazilians. Arq Bras Cardiol. 2003;80(6):635-8.

21. NHBPEP. National High Blood Pressure Education Program Working Group on High Blood Pressure in Children and Adolescent. The fourth report on the diagnosis, evaluation, end treatment of high blood pressure in children and adolescents. Pediatrics. 2004;114(2):555-76.

22. Erdreich LS, Lee ET. Use of relative operating characteristics analysis in epidemiology: a method for dealing with subjective judgement. Am J Epidemiol. 1981;114(5):649-62.

23. Coronelli CLS, Moura EC. Hipercolesterolemia em escolares e seus fatores de risco. Rev Saúde Pública. 2003;37(1):24-31.

24. Duarte JA, Ribeiro JC, Oliveira J, Mota J. The relationship between physical activity cholesterol levels in children and adolescents. Rev Bras Saúde Mater Infant. 2004;4(2):185-92.

25. Hopper C, Gruber M, Munoz K, Macconnie S, PfingstonY, Nguyen K. Relationship of blood cholesterol to body composition, physical fitness, and dietary intake measures in third grade children and their parents. Res Q Exerc Sport. 2001;72(2):182-8.

26. Stabelini Neto A, Bozza R, Ulbrich AZ, Vasconcelos IQA, Boguszewski MCS, Campos W. Fatores de risco para aterosclerose associados à aptidão cardiorrespiratória e ao IMC em adolescentes. Arq Bras Endocrinol Metab. 2008;52(6):1024-30.

27. Linhares RV, Matta MO, Lima JRP, Dantas PMS, Costa MB, Fernades Filho J. Efeitos da maturação sexual na composição corporal, nos dermatóglifos, no somatótipo e nas qualidades físicas básicas de adolescentes. Arq Bras Endocrinol Metab. 2009;53(1):47-54. 